\title{
Receptor Binding
}

National Cancer Institute

\section{Source}

National Cancer Institute. Receptor Binding. NCI Thesaurus. Code C40536.

Receptor Binding involves a temporary non-covalent, typically highly specific and high affinity, interaction through intermolecular physical forces of attraction and spatial complementarity with a diverse group of intrinsic membrane or cytoplasmic proteins that mediate the biological effects of secreted regulatory signaling molecules through modification of the activity of signal transduction pathways. 